\title{
Effects of Mach number on turbulent separation behaviours induced by blunt fin
}

\author{
S. F. Wang, Z. Y. Ren, Y. Wang
}

\begin{abstract}
An experimental study of the interaction between shock wave and turbulent boundary layer induced by blunt fin has been carried out at $M_{\infty}=7.8$ using oil flow visualization and simultaneous measurements of fluctuating wall pressure and heat transfer. This paper presents the effects of Mach number on turbulent separation behaviours induced by blunt fin.
\end{abstract}

\section{1}

\section{Introduction}

The interaction of shock wave and turbulent boundary layer induced by a hemicylindrically blunted fin has been studied extensively by many investigators. However, the majority of the work was in $M_{\infty} \leqslant 5$. Lack of a knowledge of separation behaviours in blunt fin induced hypersonic turbulent boundary layer interaction and its practical significance prompted this study.

In the study, an array of flush mounted high spatial resolution and fast response platinum film resistance thermometers and miniature high frequency pressure transducers were used to made multi-channel measurements of the fluctuating wall pressure and heat transfer on centerline upstream of the blunt fin. The surface flow patterns in the interaction region were obtained by oil flow technique. The main objective of the present experiments was to examine the primary separation line position and its correlations with diameter of blunt fin, angle of the fin and freestream Mach number in blunt fin induced turbulent boundary layer interaction. The features of shock motion in hypersonic turbulent separated flow were acquired.

\section{2}

Experimental facility, model and apparatus

The tests were conducted in the $\mathrm{JF}_{4 \mathrm{~B}}$ hypersonic free piston gun tunnel at the Institute of Mechanics, Academia Sinica. The

Received: 21 July 1996/ Accepted: 4 February 1998

S. F. Wang, Z. Y. Ren, Y. Wang

Laboratory of High Temperature Dynamics

Institute of Mechanics, Chinese Academy of Sciences

Beijing 100080, P.R. China

Correspondence to: S. F. Wang

This work was supported by National Natural Science Foundation of China. nominal test conditions were freestream Mach number of 7.8 and unit Reynolds number of $3.5 \times 10^{7} \mathrm{~m}^{-1}$. About $20 \mathrm{~ms}$ of quasi-steady uniform flow condition was provided.

The test model consisted of a flat plate of $650 \times 300 \mathrm{~mm}^{2}$ and an exchangeable blunt fin. Three fins with leading edge diameter of 10, 20 and $25 \mathrm{~mm}$ were $90 \mathrm{~mm}$ high and $200 \mathrm{~mm}$ long. The fin angles of attack were $0,10,20$ and $30^{\circ}$. The fin was mounted $450 \mathrm{~mm}$ downstream of plate leading edge. The turbulent boundary layer thickness at the fin leading edge position was about $6 \mathrm{~mm}$. The sketch of the model configuration and coordinate system is shown in Fig. 1.

Fluctuating wall pressures were measured using Kulite absolute transducers (Model XCQ $-062-25 \mathrm{~A}$ ). They were mounted along the centerline upstream of the blunt fin, flushing with the plate surface. The center-to-center spacing between two adjacent transducers was $3 \mathrm{~mm}$. The signals from the transducer were amplified by high gain amplifier $(-3 \mathrm{db}$ point of $200 \mathrm{kHz}$ ) and were then low pass filtered at $100 \mathrm{kHz}$. They were recorded in multi-channel 12 bit A/D converters. Sampling rate was set at $625 \mathrm{KHz}$. Each record length was $7 \mathrm{k}$ words.

The heat transfer sensors used for the present experiments were strip platinum film resistance thermometers. Each film was $3 \mathrm{~mm}$ long, 0.3 wide and submicro thick. They were based on a pyrex substrate and the spacing between the adjacent films were $2-4 \mathrm{~mm}$. The pyrex plate was installed in the centerline upstream of the blunt fin and was flush with the flat plate. Because the platinum thin film has negligible thermal capacity, the temperature history of the surface can be related directly to the instantaneous heat transfer by classical semi-infinite slab theory. Analog networks were used to transform the outputs of the gages to a voltage which is directly proportional to the heat transfer rate. Then signals were recorded in multi-channel 12 bit A/D converters. Sampling rate was set at $78 \mathrm{kHz}$. Each record length was $1.4 \mathrm{k}$ words.

The surface flow patterns in the interaction region including the shock induced primary separation and reattachment were obtained by the oil-dot wax paper visualization method (Wang Shifen and Wang Yu 1994).

\section{3}

\section{Results and discussions}

\section{1}

\section{Surface flow patterns}

Fig. 2 is a typical surface flow pattern in the interaction region induced by blunt fin. It can be found from Fig. 3 that the 

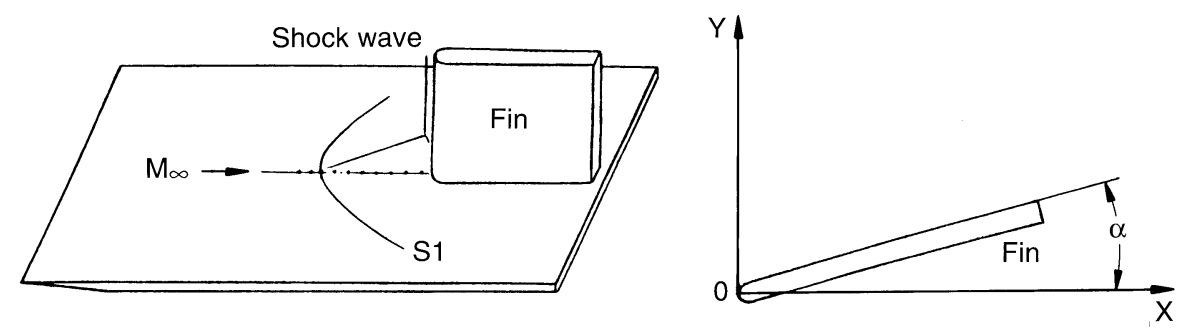
$\bar{x}$
Fig. 1. Model configuration and coordinate

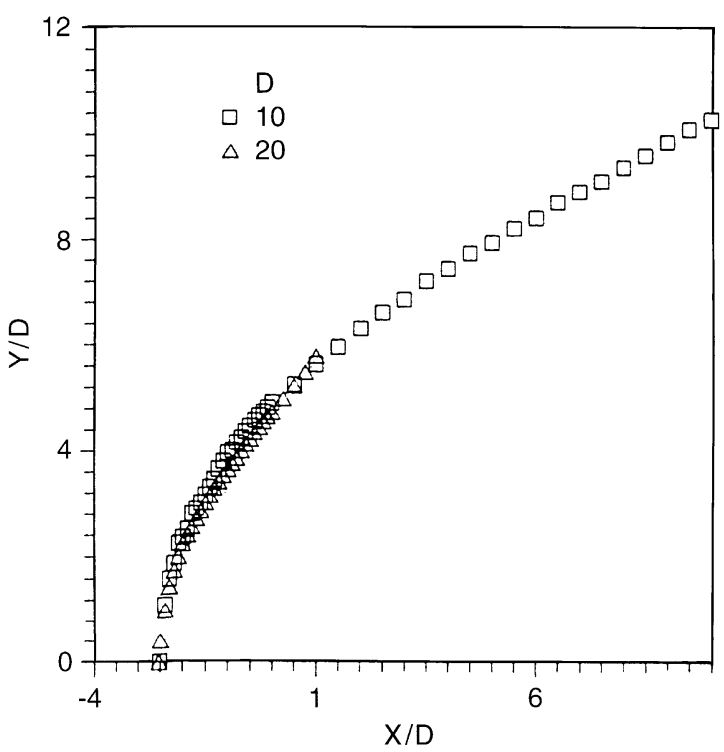

Fig. 3. Effect of bluntness on primary separation

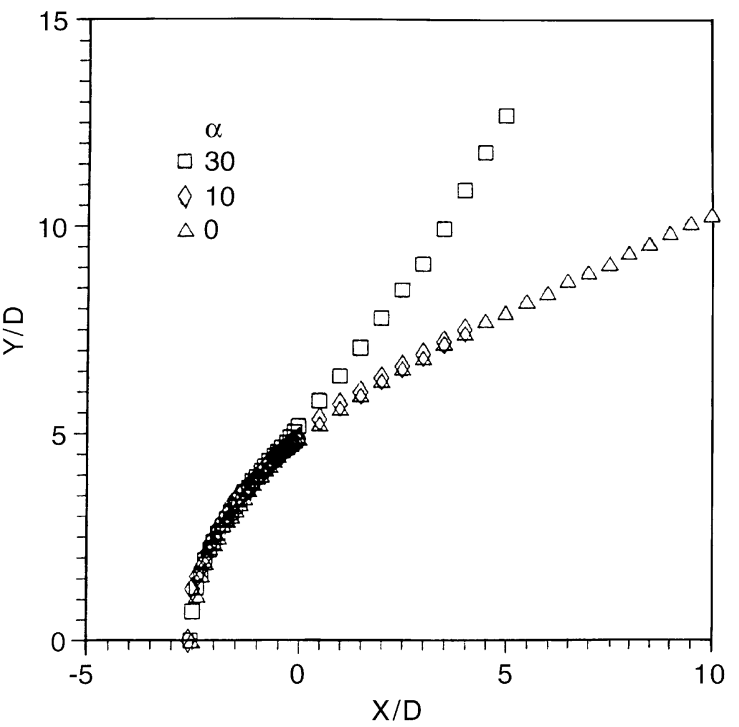

Fig. 4. Effect of fin angle on primary separation

and freestream Mach number. Because of a limited series of tests, the available data is insufficient to develop engineering correlation and prediction schemes for the boundary between the inner and outer region of the blunt fin-induced interaction. Through the comparison between the oil streak patters generated by sharp and blunt fins at $M_{\infty}=7.8$, the author divided into inner and outer region. $D$ controls the scaling in the inner region. The outer region is dependent on the fin angle 




Fig. 5. Effect of Mach number on primary separation

decided that the approximate location of the boundary, $Y / D$, is 9.5 for $\alpha=30^{\circ}$. But at low $\alpha$, it is more difficult to decide.

\section{2}

Characteristics of heat transfer and pressure signals in the interaction region

Typical samples of the time traces of the simultaneous wall pressure signals along the centerline upstream of blunt fin are shown in Fig. 6. It can be seen from the record of signals that both the instantaneous wall pressure and heat transfer are highly intermittent within the beginning interaction region, jumping randomly back and forth between the incoming undisturbed boundary layer level and a higher level induced by shock. Further downstream, the flow is disturbed for a progressively larger fraction of time. At the position of primary separation line (from surface streak patterns) the intermittent phenomena disappear. Significant intermittent phenomena due to motion of shock wave prove that hypersonic turbulent separated flow induced by blunt fin is highly unsteady.

\section{3}

Distributions of mean and standard deviations of wall pressure and heat transfer fluctuations in the interaction region

The mean wall pressure and heat transfer distributions along the centerline upstream of blunt fin are shown in Fig. 7 respectively. In front of the primary separation line mean wall pressure and heat transfer begin to increase owing to the effect of the unsteady separation shock. In the streamwise distribution two peaks are observed. The higher peaks of mean wall pressure and heat transfer are located at the attachment line. It can be seen from Fig. 7 that the streamwise distributions of mean wall pressure (heat transfer) with different diameter leading edges can be correlated by normalized with the diameter. It demonstrates again that $D$ is dominant parameter controlling the interaction scale and distribution shape of mean wall pressure and heat transfer on the centerline of the interaction surface. To the author's knowledge, the mean wall pressure and heat transfer are measured by Aso et al. (1991) in the interaction regions of shock waves and turbulent boundary

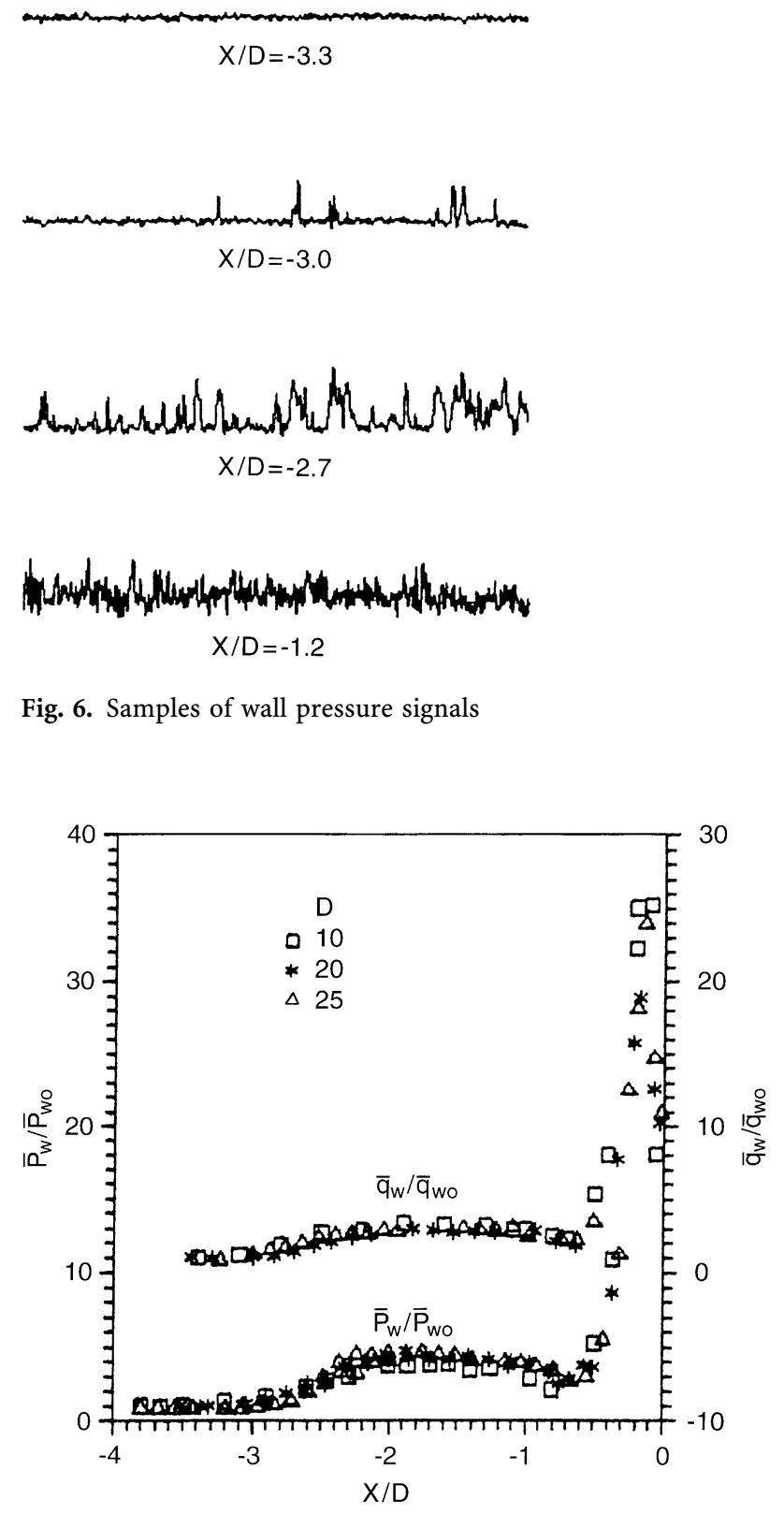

Fig. 7. Distributions of mean heat transfer and pressure along the centerline upstream of blunt fin

layers induced by blunt fins with hemicylindrical leading edges at Mach number of 4 . The data at $M_{\infty}=7.8$ shows that the higher peak pressure and heat transfer in the centerline upstream of blunt fin increase significantly compared with Aso's data, indicating that the peak pressure and heat transfer increase with Mach number. The mean wall pressure and heat transfer distributions for different Mach numbers show that $M_{\infty}$ variation alters the pressure and heat transfer levels at peaks, but has little effect on the length scale and distribution shape (Aso et al. 1991; Dolling and Bogdonoff 1982; Price and Stallings 1967; Winkelmann 1972).

Figure 8 shows the distributions of standard deviations of wall pressure and heat transfer fluctuations normalized by the local mean level along the centerline upstream of blunt fin respectively. Two peaks are observed. The first peak is located 




Fig. 8. Distributions of standard deviation of heat transfer and pressure fluctuations along the centerline upstream of blunt fin $\left(M_{\infty}=7.8, D=20 \mathrm{~mm}\right)$



Fig. 9. Separation shock foot position history $X_{s}(t)$

near primary separation line and is caused by unsteadiness in shock wave structure. In the present condition $\left(\sigma p_{w} / \bar{p}_{w}\right)_{\max }=$ 0.38 and $\left(\sigma q_{w} / \bar{q}_{w}\right)_{\max }=0.48$. At Mach 4, Aso et al. (1991) report that the maximum value of $\left(\sigma p_{w} / \bar{p}_{w}\right)$ near separation is about 0.26 , suggesting that the peak increases with Mach number.

\section{4}

\section{Separation shock unsteadiness}

The separation shock unsteadiness is characterized in terms of its position and velocity histories. Although it is difficult to measure the position of the separation shock foot directly, it is possible to bracket its location from multi-channel wall pressure (heat transfer) fluctuations (Erengil and Dolling 1992). Figure 9 shows shock foot position $X_{s}(t)$ on the centerline upstream of blunt fin with $25 \mathrm{~mm}$ diameter leading edge at the present condition. From a statistical analysis of $X_{s}(t)$ the mean location of shock foot is calculated to be $-2.75 D$. The steamwise length scale of the separation shock motion predicted from $X_{s}(t)$ is $0.85 D$ based on a $\overline{X_{s}(t)} \pm 3 \sigma X_{s}(t)$ variation. Power spectra of $X_{s}(t)$ and the fluctuating pressure

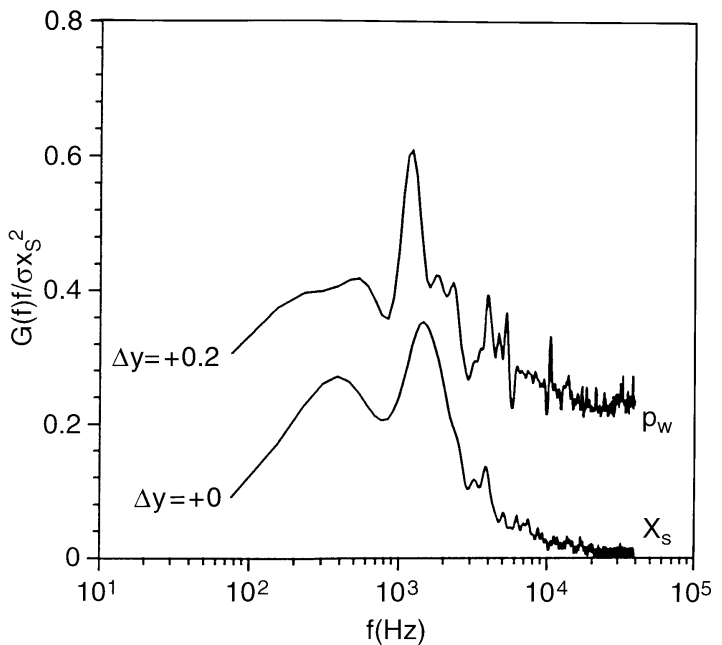

Fig. 10. Power spectra of $X_{s}(t)$ and the fluctuating pressures $P_{w}(t)$ in the shock motion region

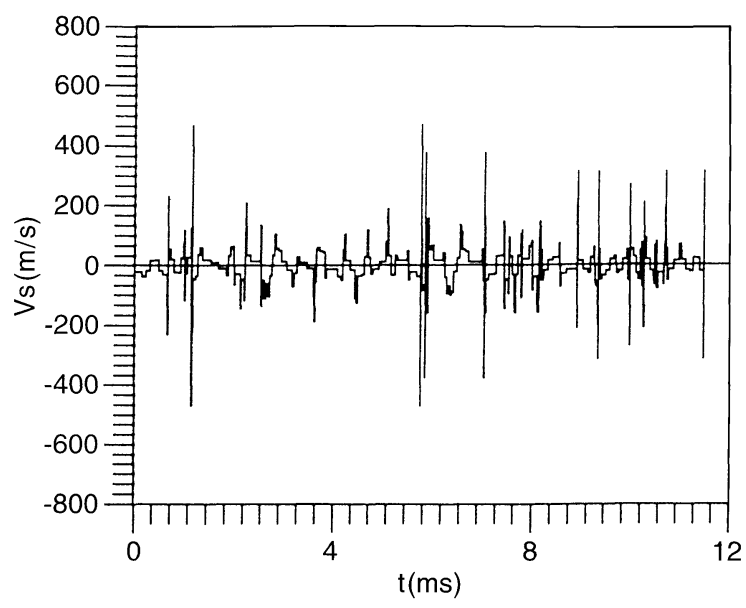

Fig. 11. Separation shock velocity $V_{s}(t)$

$P_{w}(t)$ in the shock motion region are shown in the Fig. 10. The most striking feature is the similarity of the two results for low frequencies. Two peaks at $400-500 \mathrm{~Hz}$ and $1.22-1.45 \mathrm{kHz}$ are detected for Fig. 10. The results show that the separation shock undergoes large scale and low frequency motion in front of the primary separation line.

The shock velocity $V_{s}(t)$ obtained from shock foot position is shown in Fig. 11. The mean shock speeds normalized by the freestream velocity in both the upstream and downstream directions, $\overline{\left(V_{s}\right)_{\mathrm{up}}} / V_{\infty}$ and $\overline{\left(V_{s}\right)_{\mathrm{dn}}} / V_{\infty}$, are approaching to 0.03 . Both standard deviations of the shock foot velocity normalized by the freestream velocity, $\left(\sigma V_{s}\right)_{\mathrm{up}} / V_{\infty}$ and $\left(\sigma V_{s}\right)_{\mathrm{dn}} / V_{\infty}$, are 0.031 and 0.036 respectively.

The above results are in agreement with the values at Mach 5 (Gonsalez and Dolling 1993), suggesting that $M_{\infty}$ variation has little effect on mean shock motion parameters normalized by the freestream velocity or the leading edge diameter in the centerline upstream of blunt fin. The results suggests that the unsteady phenomenon of separation shock is common to 
blowdown and gun tunnels, namely the same "physics" is at work in shock-induced turbulent separated flows in different facilities.

\section{4}

\section{Conclusions}

The oil flow visualization, measurements of wall pressure and heat transfer fluctuations in the interaction region for blunt fin and the analysis of the experimental data set lead to the following conclusions:

1. The interaction region induced by blunt fin can be divided into inner and outer region. The position and shape of the primary separation line in the inner region are mainly controlled by the leading edge diameter of the fin and the behaviours in the outer region are affected by fin angle and freestream Mach number.

2. The turbulent separation flowfield induced by blunt fin is quite unsteady. In front of the primary separation line the separation shock undergoes large scale and low frequency motion. Mach variation has little effect on mean separation shock motion parameters normalized by the freestream velocity or the leading edge diameter in the centerline upstream of blunt fin.

3. The double-peaked mean wall pressure (heat transfer) distribution exists in the centerline upstream of blunt fin. The higher peak is located at the attachment line. The characteristic double-peaked distribution of wall pressure (hear transfer) fluctuation standard deviation normalized by the local mean level also exists in the centerline upstream of blunt fin.

However, the higher peak is located near primary separation line and is caused by unsteadiness in shock wave structure. The levels at peaks increase with Mach number. The length scale and distribution shape on the centerline upstream of blunt fin are affected little by Mach number.

\section{References}

Aso S; Kuranaga S; Hayashi M (1991) Detailed measurements on unsteady properties in three-dimensional shock/turbulent boundary layer interaction induced by blunt fin at Mach number 4. AIAA Paper 91-1755

Deng Xueying; Wang Yu (1991) Separation behaviours in blunt fin induced shock wave/boundary layer interactions. Proceedings of the First International Conference on Experimental Fluid Mechanics (F.G. Zhuang Eds.), Chengdu, China, 71-76

Dolling DS; Bogdonoff SM (1982) Blunt fin-induced shock wave/turbulent boundary layer interaction. AIAA Journal 20: $1674-1680$

Erengil ME; Dolling DS (1992) Effects of sweepback on unsteady separation in Mach 5 compression ramp interactions. AIAA Paper 92-0430

Gonsalez JC; Dolling DS (1993) Correlation of interaction sweepback effects on the dynamics of shock-induced turbulent separation. AIAA Paper 93-0776

Price AE; Stallings RL (1967) Investigation of turbulent separated flows in the vicinity of fin type protuberances at supersonic Mach numbers. NASA TN-D 3804

Wang Shifen; Wang Yu (1994) A new method to obtain undistorted full-scale patter of oil flow visualization. Proceedings of the Third Asian Symposium on Visualization, Chiba, Japan, 932-937

Wirikelmann AE (1972) Experimental investigation of a fin protuberance partially immersed in a turbulent boundary layer at Mach 5 . NOLTR-72-33 